\title{
Assessment of the initiation timing of decrease in skeletal muscle mass in patients with breast cancer: A retrospective analysis

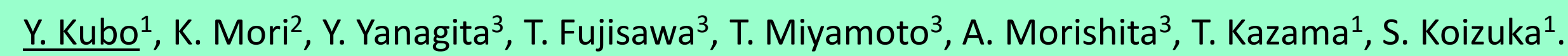

${ }^{1}$ Gunma prefectural cancer center, Palliative Medicine, Ota, Japan. ${ }^{2}$ Shizuoka Cancer Center, Clinical Research Center, Shizuoka, Japan. ${ }^{3}$ Gunma prefectural cancer center, Breast, Ota, Japan. ${ }^{4}$

\section{Introduction}

Decreased skeletal muscle mass is a poor prognostic factor in patients with cancer ${ }^{1}$-4). Therefore, determining the precise initiation timing of this decline is imperative to prevent muscle mass decrease.

\section{Objects}

This study aims to retrospectively analyze the initiation timing of muscle mass decrease in patients with breast cancer.

\section{Methods}

Among 2105 female patients with breast cancer who underwent breast cancer surgery from 2007 to 2017, we retrospectively analyzed patients under the age of 65 years who died after metastasis. We assessed the change in the skeletal muscle index (SMI) by the squared height of the skeletal muscle in Computed Tomography images at the time of diagnosis, metastasis and within 1 year of death. We considered $P=0.016$ as statistically significant

\section{Results}

\section{Figure 1. Patients selection}

2,105 female patients with breast cancer underwent surgery from 2007to 2016

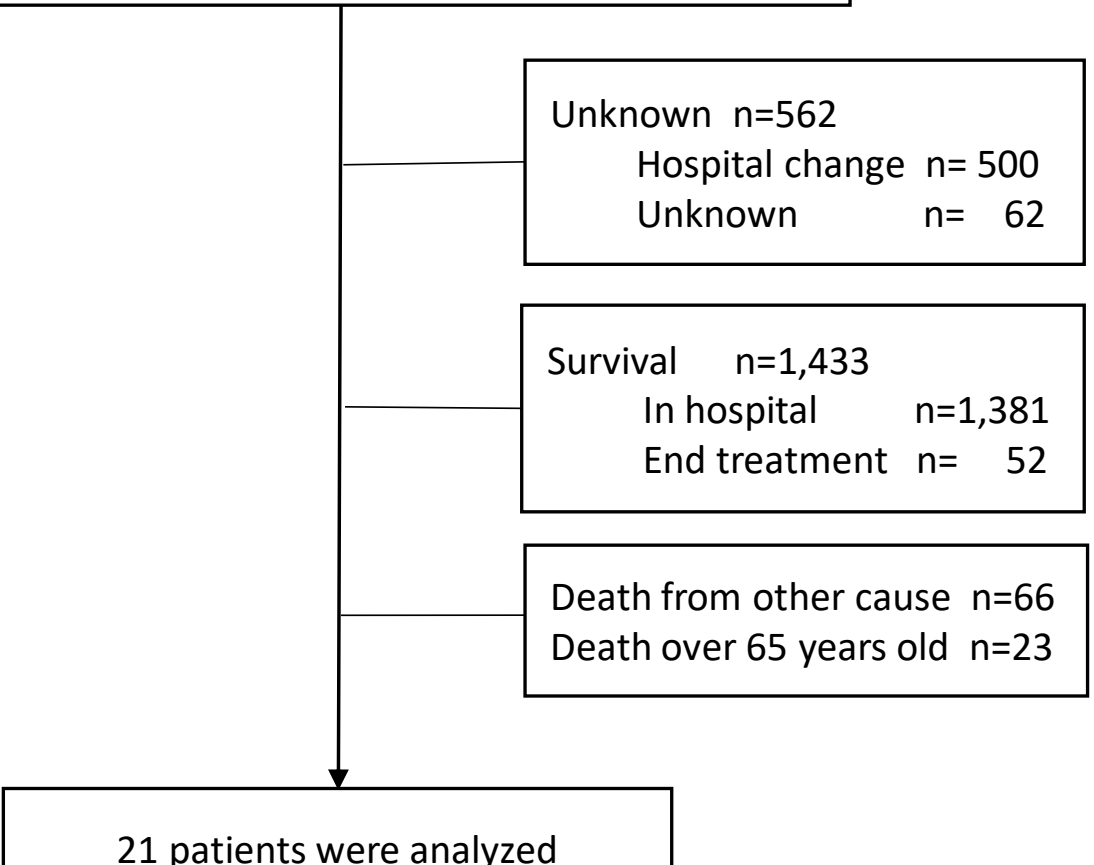

21 patients were analyzed

\section{Table 1. Patients' characteristics}

\begin{tabular}{llcc} 
& & \multicolumn{2}{c}{$(\mathrm{N}=21)$} \\
\hline $\begin{array}{l}\text { Age at surgery, (years) } \\
\text { Estrogen receptor status }\end{array}$ & \multicolumn{2}{c}{$43(30-62)$} \\
HER-2 status & Positive & 13 & $62 \%$ \\
& & & \\
Stage & Positive & 3 & $14 \%$ \\
& I & 1 & $5 \%$ \\
& II & 14 & $67 \%$ \\
Time from surgery data to metastasts, (months) & 6 & $28 \%$ \\
Time from metastasis data to death, (months) & $23(2-60)$ \\
\hline a; median (minimum to maximum) & $42(12-124)$ \\
\hline
\end{tabular}

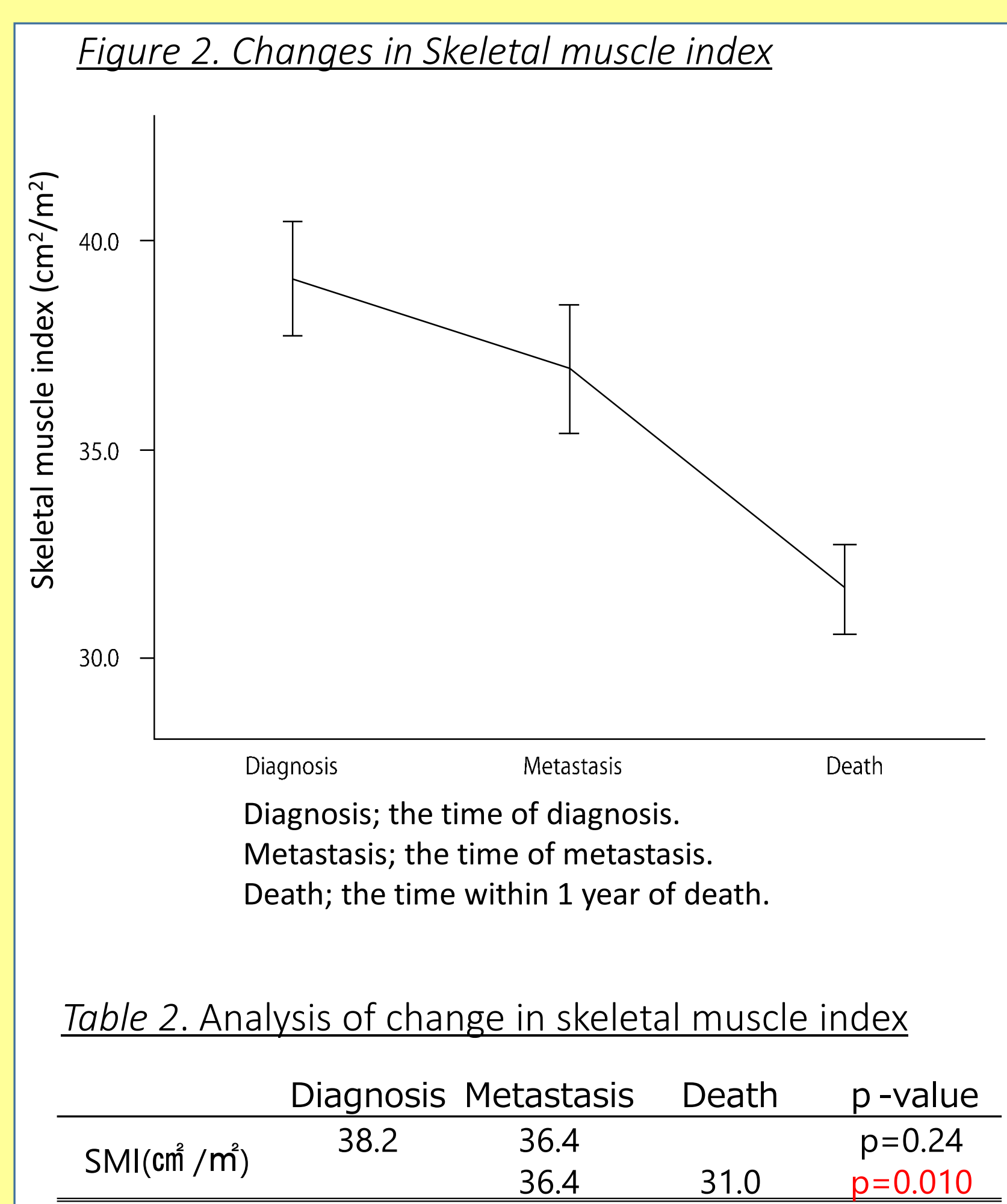

median (minimum to maximum)

38.2 (27.8-51.4), Metastasis; 36.4 (25.7-52.1), Death; 31.0 (21.0-42.3)

\section{Figure 3. CT imaged at the third lumbar vertebrae level} of one patient

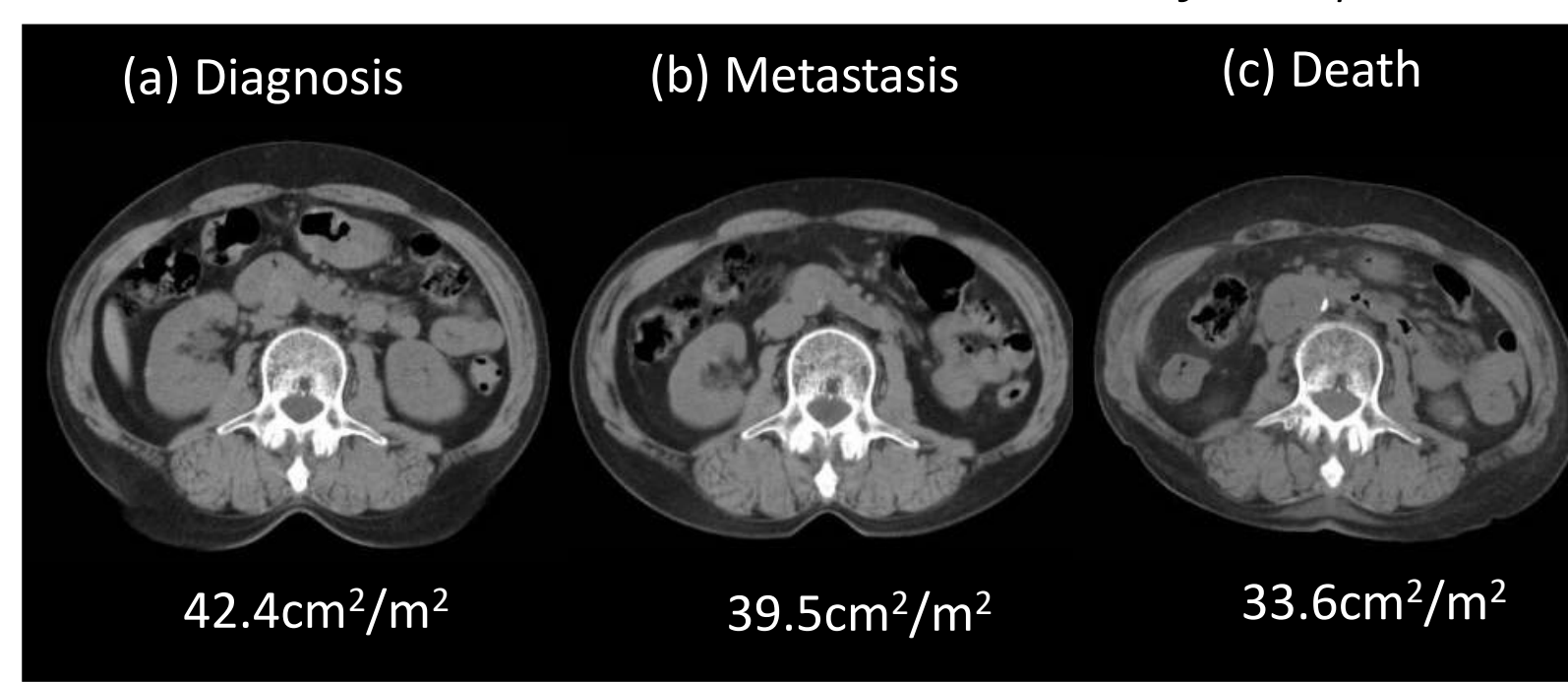

\section{Conclusion}

This study demonstrated that the skeletal muscle mass of patients with breast cancer decreases after metastasis. We believe that the findings of this study could help improve the prognosis of patients with breast cancer.

Feel free to contact me if you have any questions.

E-mail: kawabehachi@yahoo.co.jp Yoshiko KUBO 